\title{
SISDH: A Model Based on SMAs and SIRs for the Simulation of the Evolution of COVID-19 in Cameroon
}

\author{
Batchakui Bernabe1, Fandio Esdras ${ }^{2}$, Ebouky Brown², Kwate Loïc², Waffo Stephane² \\ ${ }^{1}$ Department of Computer Engineering, National Advanced School of Engineering, Yaoundé, Cameroon \\ ${ }^{2}$ Department of Computer Engineering, ENSIMAG, Grenoble, France \\ Email: esdras-emma.fandio-njylla@grenoble-inp.org, brown-wilfried.ebouky-doualla-dina@grenoble-inp.org, \\ loic.kwate-dassi@grenoble-inp.org, guy-stephane.waffo-dzuyo@grenoble-inp.org
}

How to cite this paper: Bernabe, B., Esdras, F., Brown, E., Loïc, K. and Stephane, W. (2021) SISDH: A Model Based on SMAs and SIRs for the Simulation of the Evolution of COVID-19 in Cameroon. World Journal of Engineering and Technology, 9, 527537.

https://doi.org/10.4236/wjet.2021.93035

Received: June 13, 2021

Accepted: July 27, 2021

Published: July 30, 2021

Copyright $\odot 2021$ by author(s) and Scientific Research Publishing Inc. This work is licensed under the Creative Commons Attribution International License (CC BY 4.0)

http://creativecommons.org/licenses/by/4.0/

\begin{abstract}
This paper presents a model to simulate the evolution of COVID-19 in the Cameroonian context. The presented model SISDH stands for Susceptible, Infected, Severe, Died, and Healed is made up of the mixture of a Multi-Agent System (SMA) and a SIR (Susceptible, Infected, Recovered)-based model, and mainly addresses the problem of modelling the evolution of pandemics with a high transmission rate. Multi-agent systems are used to design the SIR model's entities, namely the habitants of the region subject to the study. The experimentation carried out showed that the combination of the two concepts favours rapid decision-making. For example, the requirement to wear a mask or strict adherence to social distancing reduces the risk of spread. The application of these tough measures had theoretically leveled down the spreading of the epidemic. Besides the lowering of the number of cases when strict measures were applied, we also highlighted a significant reduction of deaths and severe illness which is a concomitant result of the lockdown. On the other hand, our experiments revealed a peak of infections a few steps after the beginning when no restrictions are made for barrier measures. The peak is followed by a sudden decrease in infection which might convey immunity of the population.
\end{abstract}

\section{Keywords}

COVID-19, SIR, SISDH, SMA, Modeling the Evolution of Pandemics

\section{Introduction}

An agent can be defined as a computer system located in an environment capa- 
ble of perceiving this environment by sensors and acting on this environment by actuators [1]. A Multi-Agent System (MAS) is a network of agents or a set made up of several agents. These agents together can interact to solve problems that are beyond the capabilities of any individual agent. For example, a group of humans can be considered as a multi-agent system where the agent is the human. It is possible to simulate the evolution of an epidemic using multi-agent systems [2]. Two main models are currently widely used to simulate the evolution of the COVID-19 pandemic: SIR (Susceptible Infected Recovered) [2] and SIS (Susceptible Infected Susceptible) [3]. The formatter will need to create these components, incorporating the applicable criteria that follow.

The SIR model gives 3 possible states of an entity in the population [4] [5]:

- Susceptible which defines that it is likely to have the virus;

- Infected which describes the state of a person infected with the virus;

- Recovered which states that the person is cured.

One drawback of this model in the case of COVID-19 modelling is that it does not take into account some important aspects such as the fact that a healed patient may be re-infected, which is not yet proven by researchers on the disease [6]. Moreover, this model does not highlight the mortality and criticality of the patient aspect in the description of the states; however, these remain very determining factors for a more real study of the evolution of COVID-19.

The SIS model [3], on the other hand, takes into account two states of an individual, namely: Susceptible and Infected. That is, a person in the population is already susceptible to having the virus; then if he has it, he becomes infected. Then, either he is healed and becomes Susceptible again, or he dies. From this description of the model, we can see that it is very unrepresentative of the reality of what the COVID 19 complex epidemic is. Concretely, this model does not already take into account the mortality aspect, we do not have a formal means of monitoring the mortality of infected individuals in our system, that said, this model does not take into account the healing and critical aspect of the patient, which undoubtedly has an impact on evolution.

From this description of the model, we can state that it is very unrepresentative concerning the complexity of an outbreak like COVID-19. This model does not already look at the mortality aspect, we do not have a formal way of tracking the mortality of infected individuals in our system, that said, this model does not consider the healing and critical state of the patient, which undoubtedly has an impact on the evolution.

In addition, it can be observed that for COVID19, other aspects should be taken into consideration, such as the wearing or not of a mask, the probability of becoming infected when one has already been infected and then cured. All this leads us to asking ourselves, what is the most appropriate model to represent the evolution of COVID 19 taking into account all its aspects and of course which one is adapted to Cameroon?

A model based on multi-agent systems, which takes into account the follow- 
ing aspects: Susceptible, Infected, Severe, Died, Healed, gives a closer and more detailed representation of the reality, allowing to determine a better evolution of the COVID 19. Such a model makes it possible to confirm the following two hypotheses:

- In a young population where the rules of containment and distancing are not applied, the number of infected people is rising sharply.

- The implementation of very strict measures strongly hinders the evolution of the COVID-19 pandemic.

The rest of this paper is made up of four main sections: a methodology, where we describe the human agent and the model we propose; an experimentation, where we validate our model; a discussion, where we compare our results with those of previous research, highlighting the strengths and weaknesses of our results; and a conclusion, where we present the perspectives of this work.

\section{Methodology}

We present in this section the construction of the model SISDH. (Susceptible, Infected, Severe, Died, Healed). SISDH is inspired by the SIR model where it adds two states (Died, Healed) and simulated the evolution of a human agent within a society made up of other human beings

\subsection{Description of the Human Agent}

The properties and methods of the human agent derive from the capacities that a real human possesses in society and which influence its state in the face of disease (Covid-19). Its properties are:

- age: integer determining the agent's age.

- wearmask: boolean variable describing whether or not an agent wears the mask.

- state: describes the state of an agent.

- Infection time: the time elapsed since the last infection of an agent.

- Severe time: time elapsed since the last critical infection of an agent.

- healed time: time elapsed since the healing of an agent.

- recovery severe time: time elapsed since the healing from severe infection.

The methods used are described as follows:

- move(): which allows the agent to move to a neighborhood area randomly and according to the probability of movement.

- status(): which allows the user to check his cur-rent status (infected, cured...). This method also calculates the time since infection (if infected), since recovery, since severe infection... and according to this and its different properties, the calculation of the probabilities of passage from one state to another is carried out.

- contact(): which is a method in which it comes into contact and infects other agents with a certain probability depending on several parameters such as the wearing of a mask and its cur-rent state 


\subsection{Model Description}

The model constitutes the environment in which the different agents will run as well as the global parameters of the agent systems. This environment has been inspired by our society in order to model as accurately as possible the different movements within a population and to observe the consequences in relation to the disease. Characteristic features of the society include the size of the population, the fact that part of the population wears masks and part does not, the fact that population movements are generally more centred on certain places (markets, schools, places of worship, etc.), the fact that when one is infected or seriously infected (hospitalised) the probability of dying or recovering varies with the passage of time, and the probability of moving also varies (a hospitalised person does not move as much as a healthy person). The properties for representing these features of society in the model are described as follows:

- population: the number of agent in the simulation.

- steps: the duration in days of simulation.

- width, height: the size of environment boundaries.

- ptrans: the probability that an infected person will transmit the disease when in contact with another agent when not wearing a mask.

- ptrans_mask: the probability of an infected person transmitting the disease when wearing a mask.

- proba_wear_mask, proba_move which represent respectively the probabilities for an agent to wear the mask and to move around.

- initial_outbreak_size initial number of infected.

- age_old which defines the age at which a staff member is considered old.

- age, agesd which represent the average and standard deviation of the age of the population.

- nb_places which represent the number of locations (randomly distributed) where agents are more likely to move.

- recovery_young_days which represents the average number of days a young person heals.

- recovery_youngsd which represents the standard deviation of the number of days a young person has been healed.

- similar parameters corresponding to the elderly.

There are also various parameters for modelling state transitions. These parameters depend on the age of the agent and the time spent (in days) since he is in his current state. This is done in order to get closer to reality, which translates into the fact that a human being has a greater or lesser chance of being infected, passing to a critical state, healing or dying depending on his age and the time he has spent in his current state. Figure 1 shows the state-transition diagram of an agent:

The following Table 1 shows the different conditions that trigger the agent's state-to-state transitions.

$\mathbf{t}$ refers to the length of time the agent has been in the given state, age represents 
(7)

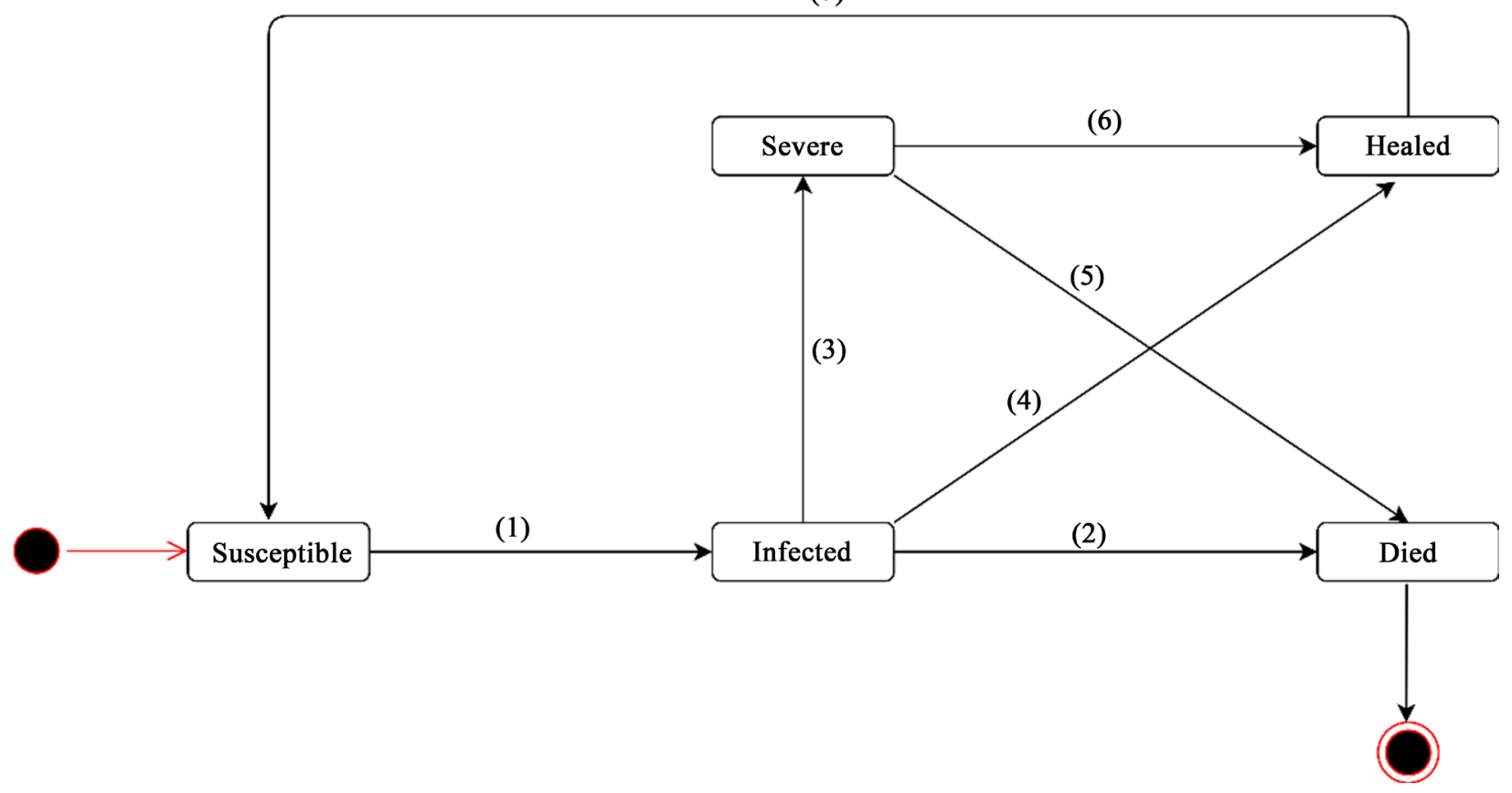

Figure 1. Transition state diagram.

Table 1. Conditions of transition of the agent from one state to another.
(1) proba $>$ ContactWithInfected (nbPersons)
(2) proba $>$ InfectedToDeath ( $t$, age)
(3) proba $>$ InfectedToSevere ( $\mathrm{t}$, age)
(4) proba $>$ InfectedToHealed ( $t$, age)
(5) proba $>$ SevereToDied (t, age)
(6) proba $>$ SevereToHealed ( $t$, age)
(7) $t>$ immunityTimeAfterHealed
(8) proba $>$ SevereToSusceptible $(t$, age)

his or her age and nbPerte number of infected persons with whom he or she has been in contact in one day.

The model therefore simulates the behaviour of the different agents during "steps" days and each evening (of the simulation), the different agents carry out an assessment in order to know their state.

\section{Experiments}

The following experiments have been implemented following [7].

We launched the environment with parameters allowing us to simulate the agents in two situations: in the containment and in the case of releasing the population. We sampled a population with 30,000 agents with an average age of 30 and distributed it in an environment with a size of $400 \times 400$, with the duration of observation extending over 365 "steps" referring to days.

It would be worth noting that the various parameters used in this experiment 
are estimated and not real and are intended to study the relevance of the model according to certain given scenarios. Also, it should be noted that in a general community, we have people of different ages but as Cameroon has a young population (median age of approximately 19), we decided to take " 30 " as our average age for the experiments.

\subsection{Release of the Population}

We tested the case where no barrier measures were complied with. We assumed that $1 \%$ of the population is initially infected and observed the evolution of the agents' status during 365 “steps”. The explicit description of the parameters is given below.

\section{1) Values of the parameters}

The previous Table 2 presents the values considered for the different parameters of the experiment.

Those values are mostly taken from the statistics observed during the pandemy whereas some of them are estimated.

The configurations used to model the transition state are described as follows:

- $\quad$ Infected to Death $(t)=0,008$

- Severely Infected to Death $(t$, age $)=\left\{\begin{array}{l}\text { age }<50:\left\{\begin{array}{l}t<10: 0.004 \times(t+1) \\ t \geq 10: 0.05\end{array}\right. \\ \text { age } \geq 50:\left\{\begin{array}{l}t<9: 0.02 \times(t+1) \\ t \geq 9: 0.2\end{array}\right.\end{array}\right.$.

- Infected to Severe $(t$, age $)=\left\{\begin{array}{l}\text { age }<50: 0.01 \\ \text { age } \geq 50: 0.2\end{array}\right.$.

- Healed to Susceptible $(t)=0.0003$.

\section{2) Results}

Afterwards implementing the model, we obtained are summarized in Figure 2.

This graph shows that in a fairly young population with a consistent number of infected people and unapplied containment and distancing rules, the number

Table 2. Parameters of the experiment.

\begin{tabular}{cc} 
population $=30,000$ & Recovery_youngs_days $=14$ \\
days $=365$ & Recovery_old_days $=21$ \\
width $=400$ & recovery_old_sd $=7$ \\
height $=400$ & recovery_severe_young_days $=7$ \\
ptrans $=0,2$ & recovery_severe_young_sd $=3$ \\
ptrans_mask $=0.03$ & age_sd $=20$ \\
age_mean $=30$ & initial_outbreak_size $=300$ \\
age_old $=50$ & proba_goto_places $=0.2$ \\
nb_places $=20$ & proba_move $=0.6$ \\
proba_wear_mask $=0.1$ & \\
\hline
\end{tabular}


of infected people increases rapidly after about 20days with a small number of severely infected people (corresponding to the number of elderly people) then falls rapidly while the number of deaths increases.

Table 3 represents the death statistics for older and younger persons.

$P_{m} / P_{T}$ and $\% m$ refer respectively to the number of deceased persons in a given category, the total number of persons in that category and the mortality rate within that category.

The following graph, represented by Figure 3, shows the repartition of the agents in the environment at the end of the simulation.

A higher density can be seen in the public places mentioned. Therefore, It can be seen that the authorization of public interactions has an influence on the number of infected people.

\subsection{Mandatory Lockdown of the Population}

We tested whether barrier measures as well as containment are imposed on the population.

\section{Parameter values}

The above parameters remain unchanged except for:

- Proba_wear_mask $=0.9$.

- Proba_move $=0.04$

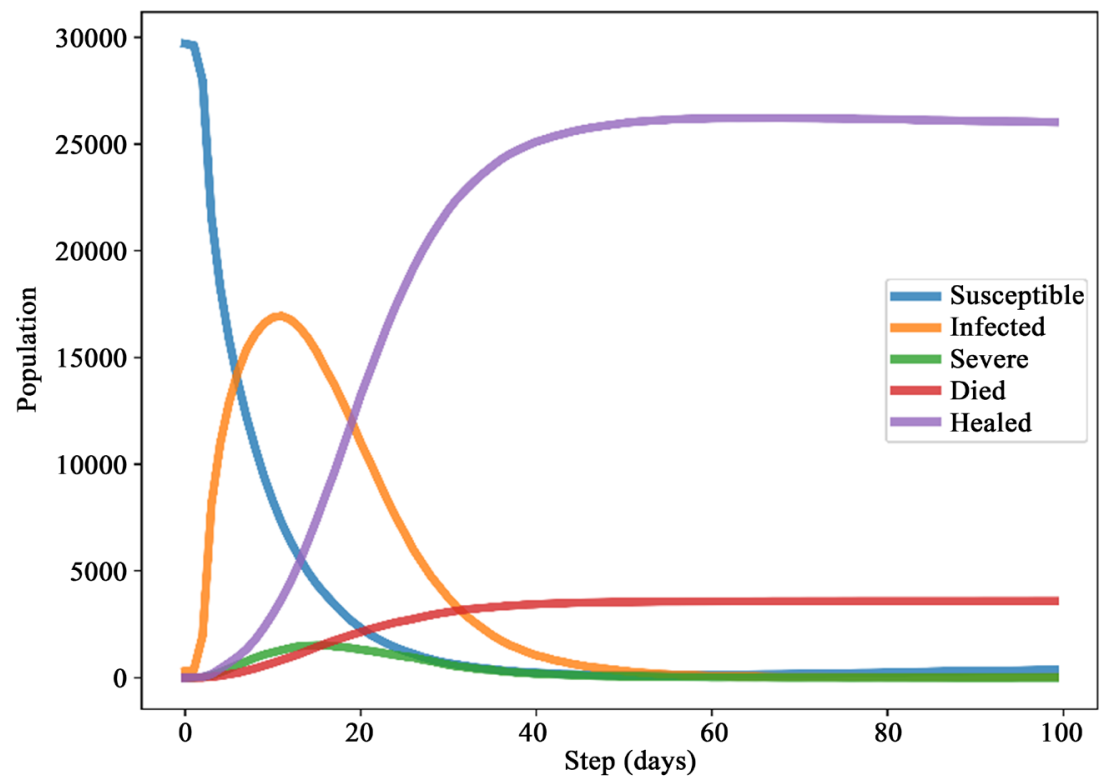

Figure 2. State agent evolution.

Table 3. Death statistics.

\begin{tabular}{ccc}
\hline Category & $\boldsymbol{P}_{m} / \boldsymbol{P}_{T}$ & $\% \boldsymbol{m}$ \\
\hline Older persons & $3427 / 4798$ & $71.42 \%$ \\
Younger Persons & $2518 / 25,202$ & $9.99 \%$ \\
Whole population & $\mathbf{5 9 4 5 / 3 0 , 0 0 0}$ & $\mathbf{1 9 . 8 2 \%}$ \\
\hline
\end{tabular}




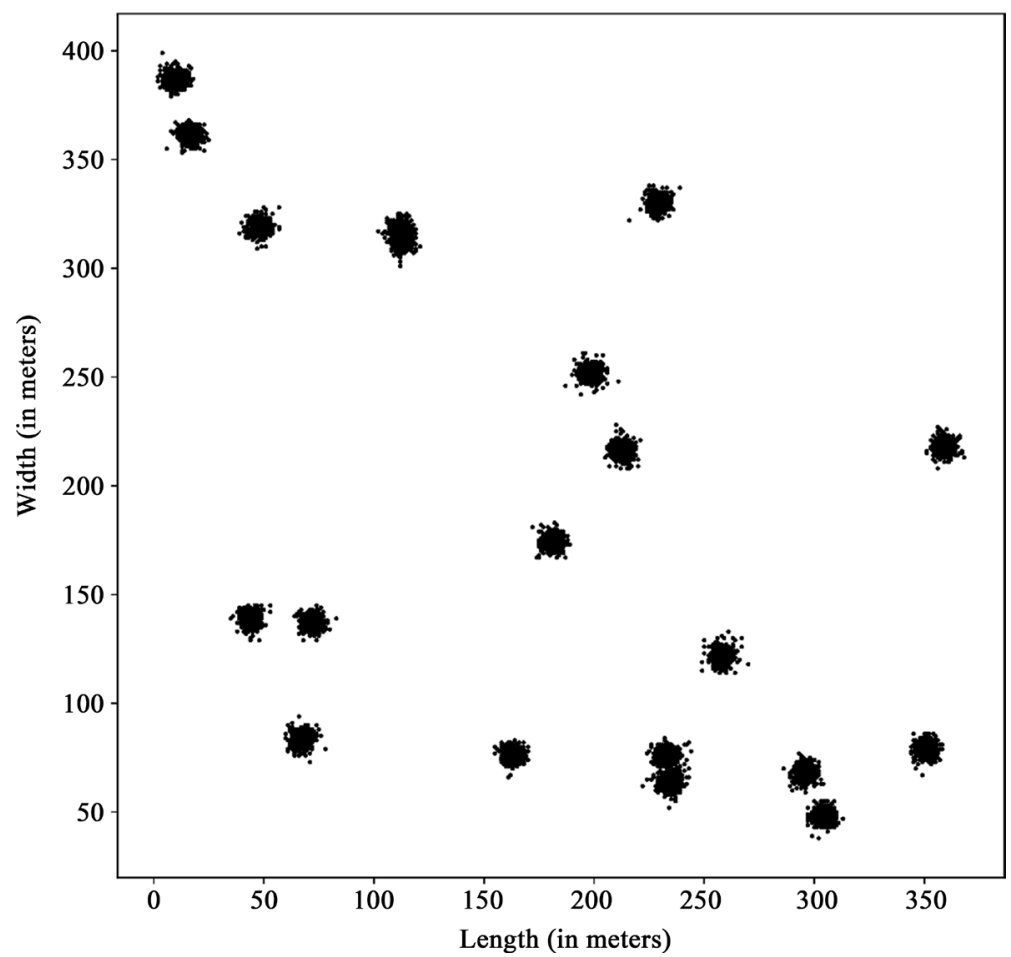

Figure 3. Distribution of the agent in the environment.

- Proba_go_to_places $=0.004$.

- steps $=365$.

We noted that the fact that the probabilities are not certain or zero just results from the fact that there is always a small proportion of the population that is recalcitrant to the law.

\section{Results}

The obtained results are described as follows. Figure 4 presents the evolution of the state of the agents.

Table 4 represents the death statistics for older and younger persons.

$P_{m} / P_{T}$ and $\% m$ represent respectively the number of deceased persons in a given category, the total number of persons in that category and the mortality rate within that category.

This curve and table show that for a fairly young population respecting the containment rules, the pandemic remains under control throughout the observation and the mortality rate is generally very low.

\section{Discussion}

Based on the various experiments that have been carried out, it appears that the behaviour of the COVID19 epidemic differs from one scenario to another:

- If one decides to implement very strict measures, one observes that the epidemic does not evolve (see Figure 4). The population is virtually unaffected by the epidemic and there are hardly any deaths or people in severe condition. 


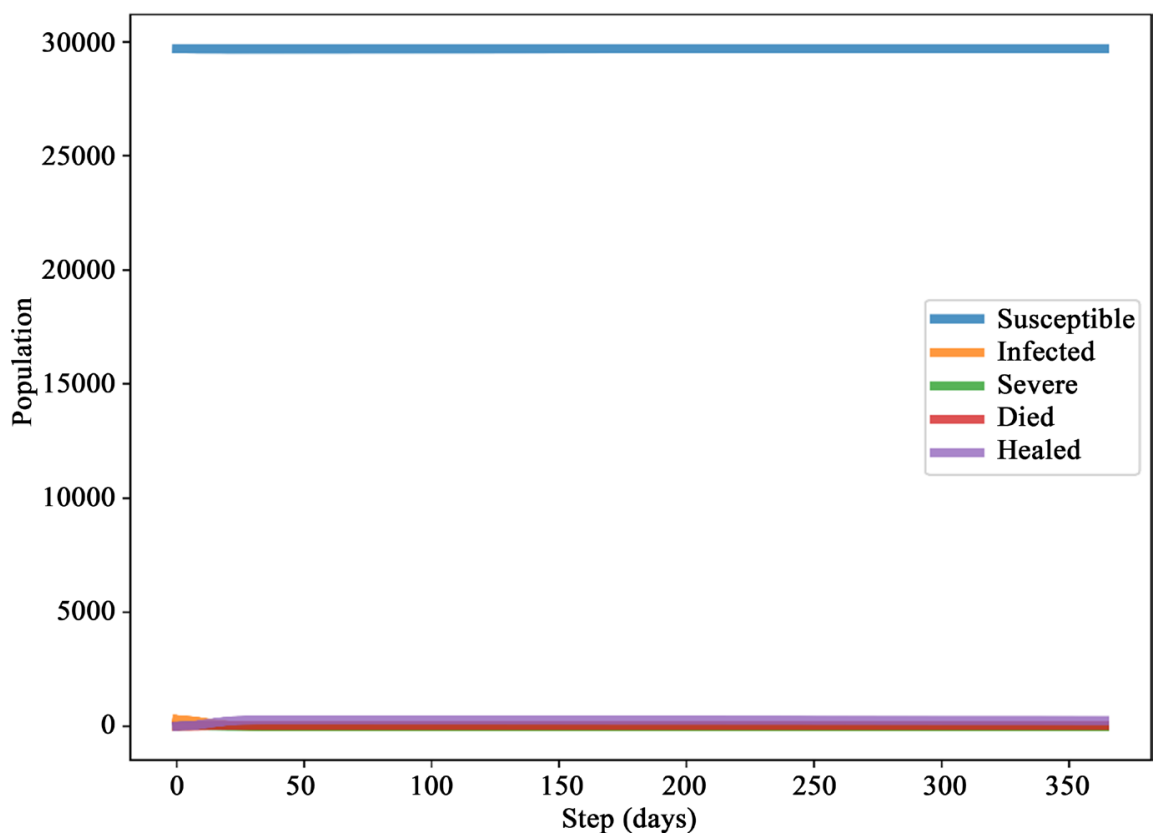

Figure 4. Evolution of the state of the agents during lockdown.

Table 4. Death statistics.

\begin{tabular}{ccc}
\hline Category & $\boldsymbol{P}_{\boldsymbol{m}} / \boldsymbol{P}_{T}$ & $\% \boldsymbol{m}$ \\
\hline Older persons & $32 / 4798$ & $0.68 \%$ \\
Younger Persons & $35 / 25,202$ & $0.14 \%$ \\
Whole population & $\mathbf{6 7 / 3 0 , 0 0 0}$ & $\mathbf{0 . 2 2 \%}$ \\
\hline
\end{tabular}

This corresponds approximately to the expected outcome of containment.

- If, on the other hand, one decides to do nothing, one can observe a large peak of infected people in the first few days, before decreasing again and stabilising. This may mean, for example, that the population that was previously infected is immunised (or it becomes more difficult for them to take the virus). Similarly, mortality peaks at about the same time as infections, before stabilising. It stabilises at a relatively high level due to the probability of death (which may depend on age) and the fact that the infections are fairly stable but continue their course. This corresponds a priori to the expected result for this scenario where the population is fairly free but retains a fairly high capacity for healing and resistance (the case of the population of Cameroon).

What can be pointed out here is that these two scenarios allow us to have a certain vision on what the consequences of their application may be. For example, the solution of no particular action (normal behaviour) can easily lead to a saturation of hospitals in the case of an increase in severe cases of the disease. This can lead to a loss of control over the evolution of the virus. On the other hand, this consideration allows business (economic aspects of the country) to continue "quite" normally, so that Cameroon will not find itself faced with major financial worries, even though hospital saturation could be the cause of many 
expenses. On the other hand, if one takes into account a total containment, one has a priori control over the evolution of the virus. On the other hand, this confinement can be quite considerable for the Cameroonian state, which will then see the economy suspended or at least very slowed down, which can be to its great disadvantage.

What can be said is to find a healthier solution that takes the advantages of the two previous methods and significantly reduces their respective drawbacks. Hence the solution of measures barriers which there-fore makes it possible to reduce the population's capacity for infection while allowing the economy to run its course at a healthier pace. This solution is still very challenging in the sense that it will require discipline both from the population but also from the Cameroonian authorities to ensure that they are respected for the good of all.

\section{Conclusion}

At the end of this article, the modelling of the evolution of COVID 19 in Cameroon using multi-agent systems was discussed. We used the SISDH model to model the epidemic after highlighting other propagation models such as SIR and SIS. The result is that this model provides a closer and more detailed representation of the reality, allowing for a better evolution of the COVID 19. However, for future research, it would be good if we could have real data (infected, healed, dead) corresponding to a locality in Cameroon, thus allowing a deeper appreciation of the effectiveness of our model. Also, the ability to have parameters (probability of passing from one state to another) validated by doctors would be of great help in this research work. In addition, it would also be interesting to be able to use GIS (Geographic Information Systems) by which one can highlight points of contamination at different levels in cities (markets, schools, bourgeois neighborhoods for example) and thus be able to experiment with evolution by taking into account these important aspects for the spread of the COVID $19 \mathrm{ep}$ idemic. It is worth noting that we did not take into account the various virus variations which appeared recently in different countries. Indeed, those have a significant impact in the evolution of the pandemy within every country including Cameroon. So, it would be nice for future research to take this into account and thus have a more robust model.

\section{Conflicts of Interest}

The authors declare no conflicts of interest regarding the publication of this paper.

\section{References}

[1] Russell, S. and Norvig, P. (1995) Artificial Intelligence: A Modern Approach. Prentice Hall, Hoboken.

[2] Ross, R. and Hamer, W. (2013) The SIR Model and the Foundations of Public Health. In: Semantic Scholar.

[3] Liu, M.X., Röst, G. and Vas, G. (2013) SIS Model on Homogeneous Networks with 
Threshold Type Delayed Contact Reduction. Computers \& Mathematics with Applications, 66, 1534-1546. https://doi.org/10.1016/j.camwa.2013.02.009 http://www.sciencedirect.com/science/article/pii/S0898122113001016

[4] David, S. and Lang, M. (2004) The SIR Model for Spread of Disease-The Differential Equation Model. MAA Publications.

[5] Kiss, I.Z., Miller, J.C. and Simon, P.L. (2017) Mathematics of Epidemics on Networks. Springer, Berlin.

[6] Tillett, R.L., Sevinksy, J.R., Hartley, P.D., et al. (2020) Genomic Evidence for Reinfection with SARS-CoV-2: A Case Study. The Lancet, 21, 52-58.

[7] https://github.com/dmnfarrell/teaching/tree/master/SIR modelling 